
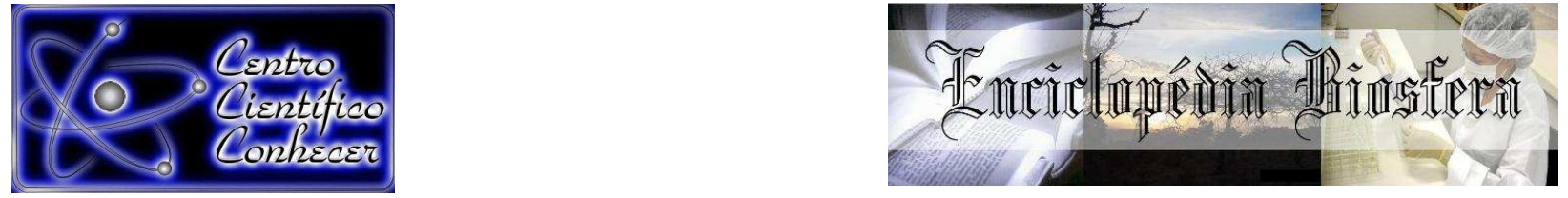

\title{
LEISHMANIOSE VISCERAL CANINA E ESTUDO DE FLEBOTOMÍNEOS APÓS CASO DE LEISHMANIOSE VISCERAL HUMANA NA CIDADE DE GOIÁS-GO
}

Thiago Souza Azeredo Bastos ${ }^{1}$; Darling Mélany de Carvalho Madrid²; Welber Daniel

Zanetti Lopes $^{3}$, Valéria de Sá Jayme ${ }^{4}$; Guido Fontgalland de Coelho Linhares ${ }^{5}$

1 Doutorando do Programa de Pós-graduação em Ciência Animal da

Universidade Federal de Goiás - UFG - Goiânia - Brasil. (tsabvet@gmail.com)

2 Mestranda do Programa de Pós-graduação em Ciência Animal da

Universidade Federal de Goiás - UFG - Goiânia - Brasil.

3 Docentes do Instituto de Patologia Tropical e Saúde Pública da Universidade Federal de Goiás - UFG - Goiânia - Brasil.

4 Docentes do Departamento de Medicina Veterinária Preventiva da Escola de Veterinária e Zootecnia da Universidade Federal de Goiás - UFG - Goiânia - Brasil.

5 Docentes do Departamento de Medicina Veterinária Preventiva da Escola de Veterinária e Zootecnia da Universidade Federal de Goiás - UFG - Goiânia - Brasil.

Recebido em: 03/10/2016 - Aprovado em: 21/11/2016 - Publicado em: 05/12/2016 DOI: 10.18677/EnciBio_2016B_060

\begin{abstract}
Durante a investigação de um foco de leishmaniose visceral humana, também se detectou leishmaniose visceral canina na cidade de Goiás-GO. Após este evento, foram instaladas armadilhas tipo CDC durante 12 meses para captura do vetor. Durante três noites seguidas ao final de cada mês, entre setembro de 2012 e agosto de 2013, instalaram-se uma armadilha dentro da residência e outra em área peridomiciliar, em um canil. No local foram capturados e identificados 304 flebotomíneos, distribuídos em quatro diferentes espécies: Nyssomyia whitmani representou $77,3 \%$ do total, enquanto Lutzomyia longipalpis, Evandromyia lenti e Nyssomyia intermedia representaram respectivamente 16,8\%, 3,9\% e 2,0\%. Após o início do estudo, apenas um flebotomíneo foi capturado dentro de um período de sete meses. Somente no mês de abril de 2013, 89,4\% do total de flebotomíneos foram capturados na armadilha instalada no peridomicílio. Em julho, capturou-se $6,5 \%$ do total e nos demais meses foram capturados menos de $1 \%$. Além de relatar o primeiro registro de leishmaniose visceral canina na cidade de Goiás, este estudo constatou que o vetor (Lutzomyia longipalpis) pode não ser capturado por período inferior a sete meses no local onde se registrou caso clínico humano e canino de leishmaniose visceral.
\end{abstract}

PALAVRAS-CHAVE: cão, Goiás, Lutzomyia. 


\title{
CANINE VISCERAL LEISHMANIASIS AND SANDFLIES FAUNA AFTER HUMAN VISCERAL LEISHMANIASIS IN GOIÁS-GO, BRAZIL
}

\begin{abstract}
During the investigation of an outbreak of registered human visceral leishmaniasis, canine visceral leishmaniasis was also detected in the city of Goiás. Shortly after diagnosis, CDC traps were installed for twelve months. During three nights sequentially, at the end of each month between September 2012 and August 2013, one trap was set inside the residence and another in a kennel in peridomiciliar area. A total of 304 sandflies were captured and identified, distributed in four different species: Nyssomyia whitmani represented $77.3 \%$, while Lutzomyia longipalpis, Evandromyia lenti and Nyssomyia intermedia accounted for respectively $16.8 \%$, $3.9 \%$ and $2.0 \%$. After the start of the study, a single sandfly was captured within a period of seven months. Only in April 2013, 89.4\% of the sandflies were caught in the trap installed outside the house. In July, $6.5 \%$ of total sandflies were captured and during the remaining months less than $1 \%$. Besides reporting the first canine visceral leishmaniasis record in the city of Goiás, this study found that the vector (Lutzomyia longipalpis) could not be captured for a period inferior to seven months at the site where human and canine visceral leishmaniasis were registered.
\end{abstract}

KEYWORDS: canine, Goiás, Lutzomyia.

\section{INTRODUÇÃO}

Flebotomíneos são insetos com considerável importância na sanidade animal e na saúde pública em diversas partes do mundo. Estes pequenos dípteros são os vetores responsáveis pela transmissão da Leishmaniose Visceral (LV) e Leishmaniose Tegumentar Americana (LTA) nas Américas (DANTAS-TORRES, 2009).

A LV, doença causada pela Leishmania (Leishmania) infantum chagasi, é uma parasitose que afeta homem e animais. Dentre os vetores comprovados desta doença estão a Lutzomyia longipalpis e Lutzomyia cruzi, entretanto, L. longipalpis é a espécie com maior distribuição em território brasileiro (QUEIROZ et al., 2012).

Por hora, a LTA é causada por distintas espécies de Leishmania (OLIVEIRA et al., 2003), sendo cada uma com ocorrência distinta em áreas geográficas diferentes. O mesmo é observado com relação aos vetores desta doença. Dentre os diversos vetores de LTA conhecidos, os dois principais são Nyssomyia whitmani e Nyssomyia intermedia, ambos possuem ampla distribuição no território brasileiro (SHIMABUKURO et al., 2011).

Segundo BRASIL (2006), quando forem diagnosticados focos de LV, uma série de procedimentos deve ser adotada para o controle da disseminação da doença. Um destes procedimentos inclui a busca por animais hospedeiros e 0 estudo entomológico.

Com o objetivo de atender tal exigência, este trabalho foi realizado para reunir informações durante a ocorrência de um foco de LV em uma pessoa que vive na zona rural em torno da cidade de Goiás.

\section{MATERIAL E MÉTODOS}

O estudo foi realizado em uma residência da zona rural, localizado há três km da região central da cidade de Goiás (1557'55.1 " latitude Sul e 5007'41.0" latitude Oeste). O município possui cerca de 23.000 habitantes, situa-se no noroeste 
do estado de Goiás, Brasil, há $135 \mathrm{~km}$ da capital estadual. Inserido em região do Bioma cerrado, possui altitude média de 512 metros, relevo bastante acidentado, com boa disponibilidade de recursos hídricos e com clima tropical úmido, sendo que a estação quente e chuvosa concentra-se entre os meses de novembro e abril (GOIÁS, 2012).

A Secretaria Municipal de Saúde em parceria com o Laboratório Central de Saúde Pública Dr. Giovanni Cysneiros (LACEN/Goiás), confirmou em agosto de 2012 um caso de Leishmaniose Visceral Humana (LVH) (SINAN, 2016). Após este registro, foram iniciadas ações de investigação entomológica conforme Manual de vigilância e controle da leishmaniose visceral (BRASIL, 2006).

Para a captura de flebotomíneos, durante 12 meses foram utilizadas duas armadilhas luminosas do tipo CDC (Center of Diseases Control), instaladas mensalmente entre as 17:00 e 07:00 horas por três noites consecutivas no intra e peridomicílio (em um canil) (BRASIL, 2006). Após a coleta, os insetos foram insensibilizados com éter e encaminhados para o Laboratório de Doenças Parasitárias do Setor de Medicina Veterinária Preventiva, da Escola de Veterinária e Zootecnia (EVZ) da Universidade Federal de Goiás (UFG) dentro de frascos plástico contendo álcool 70\%. Após triagem, em microscópio estereoscópico, os flebotomíneos foram montados conforme VILELA et al. (2003) e identificados conforme GALATI et al. (2003).

Sangue venoso (de cão suspeito) foi colhido para confirmação de leishmaniose visceral canina (LVC) usando método de diagnóstico parasitológico direto, por meio de extensão sanguínea corado com Giemsa (GOMES et al., 2016). Soro sanguíneo foi remetido ao LACEN/GO, acompanhado de identificação e da ficha de remessa de material emitida pela Secretaria de Saúde Municipal, para realização do teste rápido imunocromatográfico (TR DPP® Leishmaniose Viceral Canina - Bio Manguinhos) e ELISA (EIE - Leishmaniose Visceral Canina - Bio Manguinhos).

Os dados obtidos foram analisados por método estatístico descritivo, bem como por meio da realização do teste de Qui-quadrado (para verificar as diferenças entre as proporções de machos e fêmeas de flebotomíneos). As análises foram processadas através do software livre " $R$ " (Lucent technologies $\AA$ ). As diferenças foram consideradas significativas quando a probabilidade do erro foi inferior a $5 \%$ $(p<0,05)$.

\section{RESULTADOS E DISCUSSÃO}

Logo no início do estudo, observou-se que havia no local um cão, fêmea, com aproximadamente nove anos e que apresentava apatia, mucosas pálidas, onicogrifose, hepato-esplenomegalia e feridas nas pontas das orelhas, no dorso e no abdômen. Ainda, foi informado pelo tutor, que os sinais se iniciaram aproximadamente seis meses antes da visita (Figura 1). 

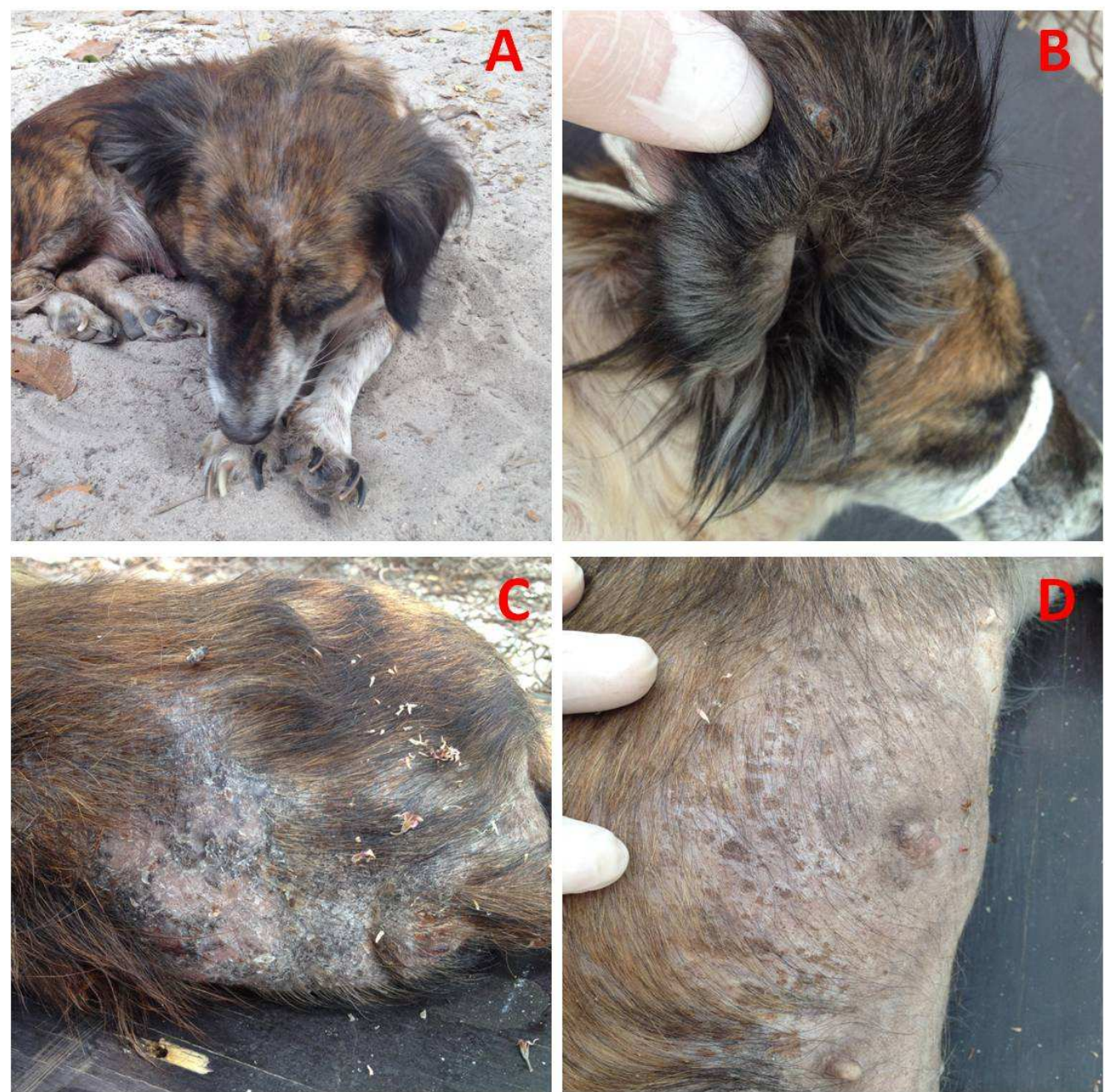

FIGURA 1: Animal apresentando sinais clínicos de Leishmaniose Visceral Canina. A: Onicogrifose; B: Lesões dérmicas na orelha; C: Lesões dérmicas no dorso; D: Lesões dérmicas no abdômen. Fonte: Arquivo pessoal.

A partir da observação deste conjunto de sinais clínicos, pode-se suspeitar de LVC conforme BRASIL (2006). Ao exame parasitológico direto, não foram observadas estruturas indicativas de leishmaniose. Mas, em ambos os testes sorológicos realizados, as amostras foram reagentes (Valor de 0,585 em relação ao Cut-Off de 0,171 para o método ELISA) e confirmaram a suspeita de leishmaniose visceral canina.

Conforme BENTES et al. (2015), o exame parasitológico direto, por ser fácil, rápido e barato, pode ser considerado como técnica de primeira escolha. Contudo, a sensibilidade é bastante variável. Neste caso, quando não forem observadas formas amastigotas de Leishmania ao exame direto, torna-se necessário a realização de outros métodos de diagnóstico, como os métodos sorológicos.

Registros anteriores relatam que a LVH ocorre na cidade de Goiás. MARTINS et al. (2002) observaram sete casos entre 1999 e 2002 e outros seis foram registrados entre 2010 e 2015 (SINAN, 2016). Deste modo, conforme estabelecido pelo Manual de vigilância e controle da leishmaniose visceral (BRASIL, 2006), a cidade de Goiás pode ser considerada como uma área de transmissão esporádica, por possuir média menor que 2,4 casos de leishmaniose Visceral nos 
últimos cinco anos. Por outro lado, este estudo registrou o primeiro caso canino diagnosticado no município.

Com relação aos flebotomíneos capturados ao longo de 12 meses (Tabela 1) no local onde se constatou os casos humano e canino de leishmaniose visceral, observou-se que todos os 304 espécimes foram capturados na armadilha instalada no peridomicílio. Além disso, nenhum flebotomíneo foi capturado nos meses de setembro, outubro, novembro, dezembro, fevereiro e março. Os maiores índices de captura ocorreram nos meses de abril $(89,47 \%)$ e julho $(6,57 \%)$. Os meses de janeiro, junho, agosto e maio somam menos de $4 \%$ do total de capturas.

TABELA 1: Espécies de flebotomíneos identificados entre setembro de 2012 e agosto de $2013 \mathrm{em}$ um foco de Leishmaniose Visceral registrado na cidade de Goiás-GO.

\begin{tabular}{|c|c|c|c|c|c|c|c|c|c|}
\hline & \multicolumn{2}{|c|}{$\begin{array}{c}\text { Nyssomyia } \\
\text { whitmani }\end{array}$} & \multicolumn{2}{|c|}{$\begin{array}{l}\text { Lutzomyia } \\
\text { longipalpis }\end{array}$} & \multicolumn{2}{|c|}{$\begin{array}{c}\text { Evandromyia } \\
\text { Lenti }\end{array}$} & \multicolumn{2}{|c|}{$\begin{array}{l}\text { Nyssomyia } \\
\text { Intermedia }\end{array}$} & TOTAL \\
\hline $\begin{array}{l}\text { Mês/Ano } \\
\text { set/2012 } \\
\text { out/2012 } \\
\text { nov/2012 } \\
\text { dez/2012 }\end{array}$ & $M$ & $\mathrm{~F}$ & M & $\mathrm{F}$ & $M$ & $\mathrm{~F}$ & $M$ & $\mathrm{~F}$ & \\
\hline $\begin{array}{r}\mathrm{jan} / 2013 \\
\mathrm{fev} / 2013 \\
\mathrm{mar} / 2013\end{array}$ & & & & & & & & 1 & 1 \\
\hline $\begin{array}{l}\mathrm{abr} / 2013 \\
\mathrm{mai} / 2013\end{array}$ & 185 & $\begin{array}{c}41 \\
3\end{array}$ & 36 & 3 & & 4 & & 3 & $\begin{array}{c}272 \\
3\end{array}$ \\
\hline jun/2013 & & & & & 4 & 1 & & 1 & 6 \\
\hline \multirow[t]{2}{*}{$\begin{array}{r}\mathrm{jul} / 2013 \\
\mathrm{ago} / 2013\end{array}$} & 6 & & 10 & 2 & $\begin{array}{l}1 \\
2\end{array}$ & & 1 & & $\begin{array}{c}20 \\
2\end{array}$ \\
\hline & 191 & 44 & 46 & 5 & 7 & 5 & 1 & 5 & \\
\hline TOTAL & & & & & & & & & 304 \\
\hline
\end{tabular}

Situação semelhante foi observada por DA PENHA et al. (2013), quando capturaram maior proporção de flebotomíneos no peridomicílio e, também, maior quantidade deste inseto após a estação chuvosa, quando o ambiente permanece quente e úmido.

Dentre as espécies identificadas, $N$. whitmani representou $77,3 \%$ do total de flebotomíneos, enquanto que $L$. longipalpis, $E$. lenti e $N$. intermedia representaram respectivamente $16,8 \%, 3,9 \%$ e $2,0 \%$. Durante todo o período do experimento, apenas 59 fêmeas $(19,4 \%)$ foram capturadas e, destas, apenas cinco $(8,4 \%)$ eram L. longipalpis. A quantidade de machos capturados foi significativamente maior $(p<0,05)$ que a quantidade de fêmeas.

Somente fêmeas são hematófagas, portanto, estas são responsáveis pela transmissão da doença e, no caso de leishmaniose visceral, apenas duas espécies foram comprovadas como vetores: L. longipalpis e L. cruzi (BRITO et al., 2014). Em contraste com outros autores, que durante um foco de LV geralmente detectam maior proporção de L. longipalpis (DA PENHA et al., 2013), durante o estudo esta foi a segunda espécie mais capturada.

Por fim, nota-se que embora o vetor desta enfermidade ( $L$. longipalpis) esteja presente na região, esta não foi a espécie predominante e nem capturada 
durante todo 0 ano. Apesar desta escassez, houve a infecção do homem possivelmente a partir do cão infectado, uma vez que é a espécie considerada reservatório desta enfermidade.

\section{CONCLUSÃO}

Este estudo relata o primeiro registro de leishmaniose visceral canina na cidade de Goiás e constatou que Lutzomyia longipalpis pode não ser capturado por período inferior a sete meses mesmo em local onde há registro de caso clínico humano e canino de leishmaniose visceral.

\section{REFERÊNCIAS}

BENTES, A. A.; RODRIGUES, D. E.; CARVALHO, E.; CARVALHO, A. L.; CAMPOS, F. A.; DE CASTRO ROMANELLI, R. M. Leishamniose tegumentar americana: um desafio diagnóstico na prática pediátrica. Revista Médica de Minas Gerais, v. 25, n. S6, 2015. doi: 10.5935/2238-3182.20150100

BRASIL, M. S. Manual de vigilância e controle da leishmaniose visceral. Brasília: Ministério da Saúde. 2006. 120p.

BRITO, V. N. D.; ALMEIDA, A. D. B. P. F.; NAKAZATO, L.; DUARTE, R.; SOUZA, C. D. O.; SOUSA, V. R. F. Phlebotomine fauna, natural infection rate and feeding habits of Lutzomyia cruzi in Jaciara, state of Mato Grosso, Brazil. Memórias do Instituto Oswaldo Cruz, v. 109, n. 7, p. 899-904, 2014. Disponível em: < http://dx.doi.org/10.1590/0074-0276140112 >. doi: 10.1590/0074-0276140112

DA PENHA, T. A.; DOS SANTOS, A. C. G.; REBÊLO, J. M. M.; MORAES, J. L. P. Fauna de flebotomíneos (Diptera: Psychodidae) em área endêmica de leishmaniose visceral canina na região metropolitana de São Luís-MA, Brasil. Biotemas, v. 26, n. 2 , p. 121-127, 2013. Disponível em: < http://dx.doi.org/10.5007/2175$\underline{7925.2013 v 26 n 2 p 121}$ >. doi: 10.5007/2175-7925.2013v26n2p121

DANTAS-TORRES, F. Canine leishmaniosis in South America. Parasites \& Vectors, v. 2, n. 1, p. 1, 2009. doi: 10.1186/1756-3305-2-S1-S1

GALATI, E. A. B.; LAINSON, R. Morfologia, terminologia de adultos e identificação dos táxons da América. In: (Ed.). Flebotomíneos no Brasil: Fiocruz, p.53-175,2003. ISBN 8575410202.

GOIÁS, P. M. D. Secretaria Municipal de Turismo e Cultura. 2012.

GOMES, A. H. D. S.; ARMELIN, I. M.; PEREIRA-CHIOCCOLA, V. L. Detecção de Leishmania (Viannia) braziliensis por PCR em amostras coletadas por raspagem de bordas de lesões de pacientes de uma área endêmica. Revista do Instituto Adolfo Lutz, v. 74, n. 4, p. 437-441, 2016. Disponível em: < http://189.126.110.61/rialutz/article/viewFile/32011/35551 >.

MARTINS, F.; SILVA, I. G. D.; BEZERRA, W. A.; MACIEL, I. J.; SILVA, H. H. G. D.; LIMA, C. G.; CANTUÁRIA, P. B.; RAMOS, O. S.; RIBEIRO, J. B.; SANTOS, A. S. Diversidade e frequência da fauna flebotomínea (Diptera: Psychodidae) em áreas 
com transmissão de leishmaniose, no Estado de Goiás. Revista de Patologia Tropical, v. 31, n. 2, p. 211-224, 2002. Disponível em: < http://dx.doi.org/10.5216/rpt.v31i2.14565 >. doi: 10.5216/rpt.v31i2.14565

OLIVEIRA, A. G. D.; ANDRADE FILHO, J. D.; FALCÃO, A. L.; BRAZIL, R. P. Estudo de flebotomíneos (Diptera, Psychodidae, Phlebotominae) na zona urbana da cidade de Campo Grande, Mato Grosso do Sul, Brasil, 1999-2000. Cadernos de Saúde Pública, v. 19, n. 4, p. 933-944, 2003. Disponível em: < http://dx.doi.org/10.1590/S0102-311X2003000400016 >. doi: 10.1590/S0102311X2003000400016

QUEIROZ, M. F. M.; VARJÃO, J. R.; MORAES, S. C. D.; SALCEDO, G. E. Analysis of sandflies (Diptera: Psychodidae) in Barra do Garças, State of Mato Grosso, Brazil, and the influence of environmental variables on the vector density of Lutzomyia longipalpis (Lutz \& Neiva, 1912). Revista da Sociedade Brasileira de Medicina Tropical, v. 45, n. 3, p. 313-317, 2012. Disponível em: < http://dx.doi.org/10.1590/S0037-86822012000300007 >. doi: 10.1590/S003786822012000300007

SHIMABUKURO, P. H. F.; GALATI, E. A. B. Lista de espécies de Phlebotominae (Diptera, Psychodidae) do Estado de São Paulo, Brasil, com comentários sobre sua distribuição geográfica. Biota Neotropica, v. 11, n. 1a, p. 1-20, 2011. Disponível em: $<$ http://www.biotaneotropica.org.br/v11n1a/en/abstract?inventory+bn0361101a2011 $>$.

SINAN. Sistema de Informação de Agravos de Notificação [online]. Brasilia, Brasil. 2016. [capturado 12 set. 2016] Disponível em: http://portalsinan.saude.gov.br/dados-epidemiologicos-sinan.

VILELA, M. L.; RANGEL, E. F.; LAINSON, R. Métodos de coleta e preservação de flebotomíneos. In: (Ed.). Flebotomíneos no Brasil: Fiocruz, p.353-367.2003. 